\title{
Influence of Sm and Yb Pollution on Superconducting Properties of YBCO Bulk Superconductors
}

\author{
D. Volochová ${ }^{a, *}, \mathrm{~K}$. JureK ${ }^{b}, \mathrm{M}$. Radušovská ${ }^{a}, \mathrm{~S}$. PiovarČI ${ }^{a}$, V. $\operatorname{Antal}^{a}$, \\ J. KOVÁC $\check{C}^{a}$, M. JIRSA ${ }^{b}$ AND P. DIKO ${ }^{a}$ \\ ${ }^{a}$ Institute of Experimental Physics SAS, Watsonova 47, 04001 Košice, Slovak Republic \\ ${ }^{b}$ Institute of Physics ASCR, Na Slovance 2, CZ-182 21 Prague 8, Czech Republic
}

\begin{abstract}
$\mathrm{YBa}_{2} \mathrm{Cu}_{3} \mathrm{O}_{7-\delta}$ (YBCO, Y-123) bulk superconductors with a nominal composition $\mathrm{Y}_{1.5} \mathrm{Ba}_{2} \mathrm{Cu}_{3} \mathrm{O}_{x}$ and 1 wt\% $\mathrm{CeO}_{2}$ addition were prepared by the Top Seeded Melt Growth (TSMG) process. Small single-crystalline pieces, cut from the $\mathrm{SmBa}_{2} \mathrm{Cu}_{3} \mathrm{O}_{y}$ (Sm-123) bulk, were used for seeding of epitaxial growth. Wavelength-dispersive spectrometry (WDS) confirmed the presence of $0.1 \mathrm{wt} \% \mathrm{Sm}$ from the seed and $0.25 \mathrm{wt} \% \mathrm{Yb}$ from the substrate, practically in the whole sample volume. The influence of this $\mathrm{Sm}$ and $\mathrm{Yb}$ contamination on superconducting properties of grown bulk materials is reported.
\end{abstract}

DOI: 10.12693/APhysPolA.126.358

PACS: 74.60.Bf, 74.72.-h, 81.40.Rs, 74.25.Ha, 74.81.Bd, 74.25.Op

\section{Introduction}

The top seeded melt growth (TSMG) process is known to be one of the most effective ways to fabricate large single-grain $\mathrm{YBa}_{2} \mathrm{Cu}_{3} \mathrm{O}_{7-\delta}$ ( $\left.\mathrm{YBCO}, \mathrm{Y}-123\right)$ bulk superconductors [1]. These superconductors are commonly grown using either $\mathrm{SmBa}_{2} \mathrm{Cu}_{3} \mathrm{O}_{y}(\mathrm{Sm}-123)\left(T_{p} \approx\right.$ $\left.1060{ }^{\circ} \mathrm{C}\right)$ or $\mathrm{NdBa}_{2} \mathrm{Cu}_{3} \mathrm{O}_{z}(\mathrm{Nd}-123)\left(T_{p} \approx 1070{ }^{\circ} \mathrm{C}\right)$ seed crystals [2] due to their higher peritectic temperature, $T_{p}$ or thin film seeds [3]. A processing window for the growth of Y-123 single-crystals $\left(T_{p} \approx 1010{ }^{\circ} \mathrm{C}\right)$ becomes sufficiently large to decompose the YBCO pellet without melting the seed. Nevertheless, Sm-123 seed dissolution has been reported [4], which leads to a diffusion of Sm into the sample volume.

Additional source of contamination may come from a substrate used for YBCO pellet. It has already been shown, that melt processed YBCO samples, grown on the $\mathrm{Al}_{2} \mathrm{O}_{3}$ substrate, contain $\mathrm{Al}$ due to its dissolution in the melt [5].

We report on contamination of YBCO bulk superconductors by $\mathrm{Sm}$ from the seed and by $\mathrm{Yb}$ from the used substrate. The influence of this contamination on superconducting properties of prepared samples is shown.

\section{Experimental}

YBCO single-grain bulk superconductors with a nominal composition $\mathrm{Y}_{1.5} \mathrm{Ba}_{2} \mathrm{Cu}_{3} \mathrm{O}_{x}$ and $1 \mathrm{wt} \% \mathrm{CeO}_{2}$ addition were fabricated by the Top Seeded Melt Growth (TSMG) process. For the TSMG process the Sm-123 or Nd-123 single crystals were used as nucleation seeds. To prevent pollution of the sample, $\mathrm{Y}_{2} \mathrm{BaCuO}_{5}$ buffer layers (Y-211 BL, $\varnothing 5 \mathrm{~mm}$, thickness $3 \mathrm{~mm}$ ) were placed between the seed and the pellet [6] and the pellet was placed on a thin layer of $\mathrm{Yb}_{2} \mathrm{O}_{3}+\mathrm{Y}-123$ powder mixture $(60: 40 \mathrm{wt} \%)$, as a substrate in order to prevent undesirable nucleation from the bottom. Later, three different

\footnotetext{
*corresponding author; e-mail: volochova@saske.sk
}

kinds of substrates were tested: $\mathrm{Yb}_{2} \mathrm{O}_{3}$ substrate, $\mathrm{Y}-211$ and $\mathrm{Yb}_{2} \mathrm{O}_{3}$ substrate and $\mathrm{Y}_{2} \mathrm{O}_{3}$ and $\mathrm{Yb}_{2} \mathrm{O}_{3}$ substrate. The samples were treated in a chamber furnace with the time/temperature profile optimized for high Y-123 crystal quality [7].

The trapped field distribution of the bulk samples oxygenated at $400{ }^{\circ} \mathrm{C}$ for $200 \mathrm{~h}$ was mapped by the Hall probe scanning after magnetization in $1.4 \mathrm{~T}$ field, applied parallel to the c-axis in liquid nitrogen.

Small samples for oxygenation process, performed at $400{ }^{\circ} \mathrm{C}$ for 150 hours in a flowing oxygen atmosphere, and for magnetization measurements had a shape of a slab with the dimensions $2 \times 2 \times 1 \mathrm{~mm}^{3}$. The smallest dimension was parallel to the $c$-axis of the crystal.

The magnetization measurements were done in a vibrating-sample magnetometer (VSM) with magnetic fields up to $6 \mathrm{~T}$, applied parallel to the $c$-axis of the crystal, at the temperature of $77 \mathrm{~K}$. The critical current densities, $J_{c}$, were calculated using the extended Bean model [8] for rectangular samples. The critical transition temperatures, $T_{c}$, were determined from the magnetic transition curves at $50 \%$ of the low temperature magnetization. The magnetizations were measured in an applied external magnetic field of $2 \mathrm{mT}$ after zero field cooling.

\section{Results and discussion}

Magnetization measurements of the YBCO singlegrain sample prepared by the optimized TSMG process $\left(T_{\max }=1040^{\circ} \mathrm{C} / 10 \mathrm{~h}\right)[7]$ revealed relatively low critical temperature, $T_{c}(50 \%)=84.7 \mathrm{~K}$ (Fig. 1a). Additionally, peak effect was observed in the field dependence of critical current density, $J_{c}$ (Fig. 1b). These observations are usually typical for chemically doped samples, where impurity atoms substitute atoms in the crystal lattice of superconductor, suppress superconductivity locally and can act as additional pinning centers [9]. Therefore, we analyzed the concentration of possible elements, which could pollute the sample during melt processing, by WDS line microanalyses. Sm from the seed and $\mathrm{Yb}$ from the substrate were identified in concentrations $0.1 \mathrm{wt} \%$ and 
$0.25 \mathrm{wt} \%$, respectively, practically in the whole sample volume.
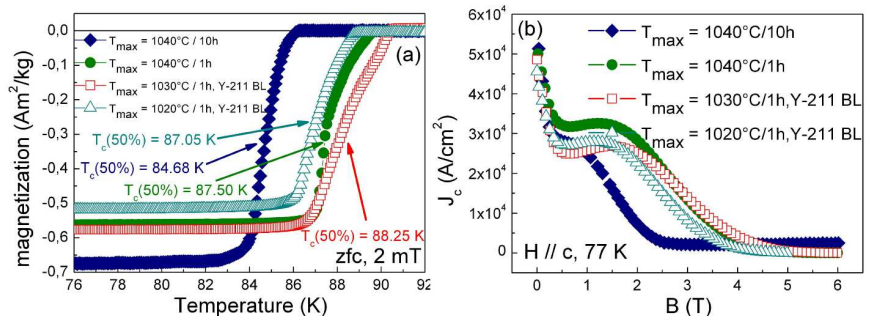

Fig. 1. Magnetization measurements of Y-123 sample prepared by the TSMG process with different $T_{\max }$ using a Sm-123 seed and $\mathrm{Yb}_{2} \mathrm{O}_{3}+\mathrm{Y}-123$ substrate.
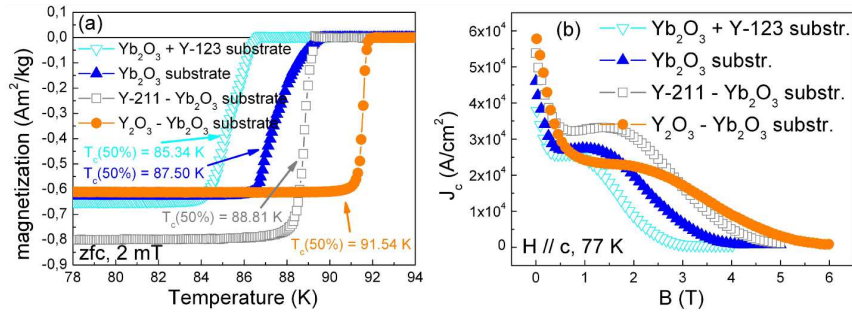

Fig. 2. Magnetization measurements of Y-123 samples grown using different kinds of substrates. Nd-123 singlecrystals were used as seeds in this case.

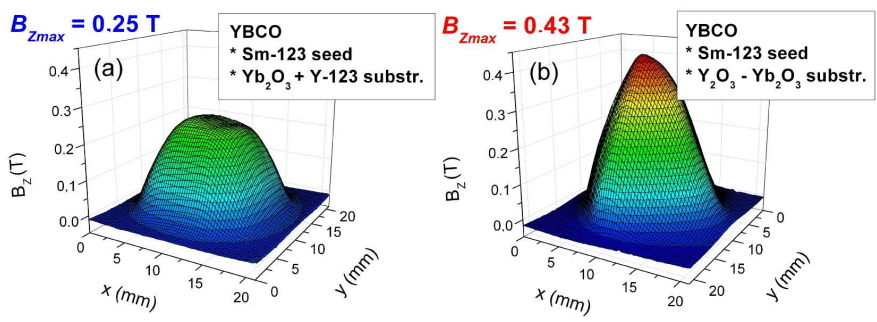

Fig. 3. Trapped magnetic field profiles of YBCO samples prepared using Sm-123 seeds and different kinds of substrates.

Contamination of YBCO samples by Sm has already been reported [6]. Dissolution of the Sm-123 seed used during TSMG melt processing of Y-123 was observed. It was supposed that at high temperatures, the seed/melt interface is thermodynamically unstable, which results in partial or full dissolution of seed during melt processing. In order to minimize this Sm pollution, we performed several experiments. First, we decreased the holding time on $T_{\max }$ to $1 \mathrm{~h}$. Nevertheless, the critical temperature was found to be still low and pronounced peak effect was present in $J_{c}(B)$ dependence (Fig. 1). We obtained similar results even when we decreased $T_{\max }$ $\left(T_{\max }=1030^{\circ} \mathrm{C}\right.$ or $\left.1020^{\circ} \mathrm{C}\right)$ and used Y-211 buffer layer (Y-211 BL, $\varnothing 5 \mathrm{~mm}$, thickness $3 \mathrm{~mm}$ ) as a barrier for $\mathrm{Sm}$ diffusion [6].

In order to minimize contamination of YBCO samples by $\mathrm{Yb}$, different kinds of substrates were tested. In these experiments, Nd-123 single-crystals were used as the nucleation seed. It can be seen in Fig. 2, that $\mathrm{Y}_{2} \mathrm{O}_{3}$ and $\mathrm{Yb}_{2} \mathrm{O}_{3}$ substrates lead to high $T_{c}$ and sharp transition to normal state $\left(T_{c}(50 \%)=91.5 \mathrm{~K}, T_{c}^{\text {onset }}=92.04 \mathrm{~K}\right.$, $\left.\Delta T_{c}=0.45 \mathrm{~K}\right)$. In this case the pronounced peak effect was suppressed.

The trapped magnetic field profile of this YBCO sample is shown in Fig. 3b. It can be compared with trapped magnetic field profile of the YBCO sample, which is contaminated by $\mathrm{Yb}$ from the substrate (Fig. 3a). The increase of the maximum trapped field, $B_{Z \max }$ can be clearly recognized.

\section{Conclusions}

YBCO single-grain bulk superconductors were prepared by the optimized TSMG process. WDS line microanalyses confirmed that prepared samples contain besides $0.1 \mathrm{wt} \% \mathrm{Sm}$ from the seed, also $0.25 \mathrm{wt} \% \mathrm{Yb}$ from the substrate. This contamination significantly influenced their superconducting properties. Low critical temperature, $T_{c}$ of prepared samples and peak effect in their $J_{c}(B)$ dependences were observed. Decrease of dwell time on $T_{\max }=1040{ }^{\circ} \mathrm{C}$ to $1 \mathrm{~h}$, using a $\mathrm{Nd}-123$ seed and a combination of $\mathrm{Y}_{2} \mathrm{O}_{3}$ and $\mathrm{Yb}_{2} \mathrm{O}_{3}$ substrate, led to a high critical temperature with a sharp transition to the normal state of prepared sample. In this case the pronounced peak effect was suppressed and higher maximum trapped magnetic field was reached.

\section{Acknowledgments}

This work was realized within the Framework of the projects: ITMS 26220120019, ITMS 26220120035, ITMS 26220220061, ITMS 26220220041, ITMS 26110230061, VEGA project No. 2/0090/13 and SAS Centre of Excellence: CFNT MVEP.

\section{References}

[1] M. Morita, S. Takebayashi, M. Tanaka, K. Kimura, K. Miyamoto, K. Savano, Adv. Supercond. 3, 733 (1991).

[2] D.A Cardwell, N. Hari Babu, Physica C 445, 1 (2006).

[3] T.Y. Li, C.L. Wang, L.J. Sun, S.B. Yan, L. Cheng, X. Yao, J. Xiong, B.W. Tao, J.Q. Feng, X.Y. Xu, C.S. Li, D.A. Cardwell, J. Appl. Phys. 108, 023914 (2010).

[4] Y.A. Jee, G.W. Hong, C.J. Kim, T.H. Sung, Supercond. Sci. Technol. 11, 650 (1998).

[5] P. Diko, G. Stöver, G. Krabbes, Supercond. Sci. Technol. 15, 217 (2002).

[6] C.J. Kim, J.H. Lee, S.D. Park, B.H. Jun, S.C. Han, Y.H. Han, Supercond. Sci. Technol. 24, 015008 (2011).

[7] D. Volochová, P. Diko, M. Radušovská, V. Antal, S. Piovarči, K. Zmorayová, M. Šefčiková, J. of Cryst. Growth 353, 31 (2012).

[8] C.P. Bean, Phys. Rev. Lett. 8, 250 (1962).

[9] L. Shlyk, G. Krabbes, G. Fuchs, G. Stöver, S. Gruss, K. Nenkov, Physica C 377, 437 (2002). 